\title{
Design and Implementation of the Evaluation System of the Theatre
}

\author{
Wei Jiang ${ }^{[1,2,3]}$, Xiru Guan ${ }^{[1,2,3]}$, Yujian Jiang ${ }^{[1,2,3]}$, Shaobo Wang ${ }^{[1,2,3]}$ \\ ${ }^{1}$ Information Engineering School, Communication University of China \\ ${ }^{2}$ Key Laboratory of Acoustic Visual Technology and Intelligent Control System, Ministry of Culture \\ ${ }^{3}$ Beijing Key Laboratory of Modern Entertainment Technology \\ Beijing, China \\ aemail:13261095120@163.com
}

Keywords: Theater Evaluation; Web Technology; Bootstrap; AHP (Analytic Hierarchy Process)

\begin{abstract}
The evaluation system of the theater is based on user data, professional evaluation and audience comments, and we use web technology to build the system. In this paper, the framework, function and technical design of the evaluation system of the theatre are presented. Thebootstrap is used to buildthe framework. Apache and MySQL are used in the server database.Through a variety of technical methods, we ultimately can establish a theater evaluation system which has the functions of user classification, grading, information browsing and management.
\end{abstract}

\section{Introduction}

With the rapid development of China's economy, people's income and living standards continue to improve, at the same time, they pay more attention to the construction of spirit. Drama, opera, concerts, concerts and other diverse forms of performance are increasingly becoming popular culture consumption projects. The theatre is dedicated to performing drama, opera, dance, music and other entertainments, but also an important carrier of cultural and artistic performances. It undertakes the social responsibility of the national culture and art education, which leads and promotes the quality of the national culture, and represents the cultural dignity of a country and a nation. A classic theatre is a beautiful card, and it can become a national landmark. Vienna is famous for its golden hall. Sydney Opera House also let the country sparkling.[1]The National Theatre has become a symbol of the theater in our country.

In recent years, a large number of theatres have sprung up. However, many theaters took the large scale and the shape of the building as the target, and this will produce a lot of problems. The blind pursuit of the scale of construction often neglects its use value and economic benefits. In some places, the construction of the theater is of low quality, which is completely unequalto the local cultural consumption level.On the other hand,the operation of the theater is extremely short of support from the audience.As a consequence, it will lead to many problems, such as less performance and low attendance. It has caused a great waste of resources to a large extent. The audience is quite blind in the choice of cultural consumption.

The sources of these problems exist in many aspects.On the one hand, the construction of the theater has no consistent standards. On the other hand, the lack of a large number of professional personnel evaluation and audience real-time feedback will lead that the construction and operation of the theater cannot be a good fit, and it could not meet the needs of consumers. The current Internet technology provides more choices for the performance and operation. According to the users' habits data, professional evaluation and comment on the audience, we establish the evaluation system of the theater so that we can provide consumer reference for the audience. We can provide the industry benchmark for the builders and designers. And we can also provide operators with operational and reform indicators. Itcan promote the construction and operation of the theater with good specification and high quality. 


\section{System Requirements Analysis}

The system contains three different levels of user types depending on the user's permissions and functional requirements.

\section{A. Ordinary audience}

After ordinary audience logs on the site, the basic process is shown in Figure 1.At first, this type of user can query the theater information, includingthe introduction of the theater,evaluation informationand other basic information. Besides, users can evaluate the theater. They evaluate four aspects: overall evaluation, performance evaluation, service evaluation, and environmental assessment.Moreover, users can put forward suggestions and opinions on the theatre by description in words.In addition, users can also query the latest play, star style and other information.

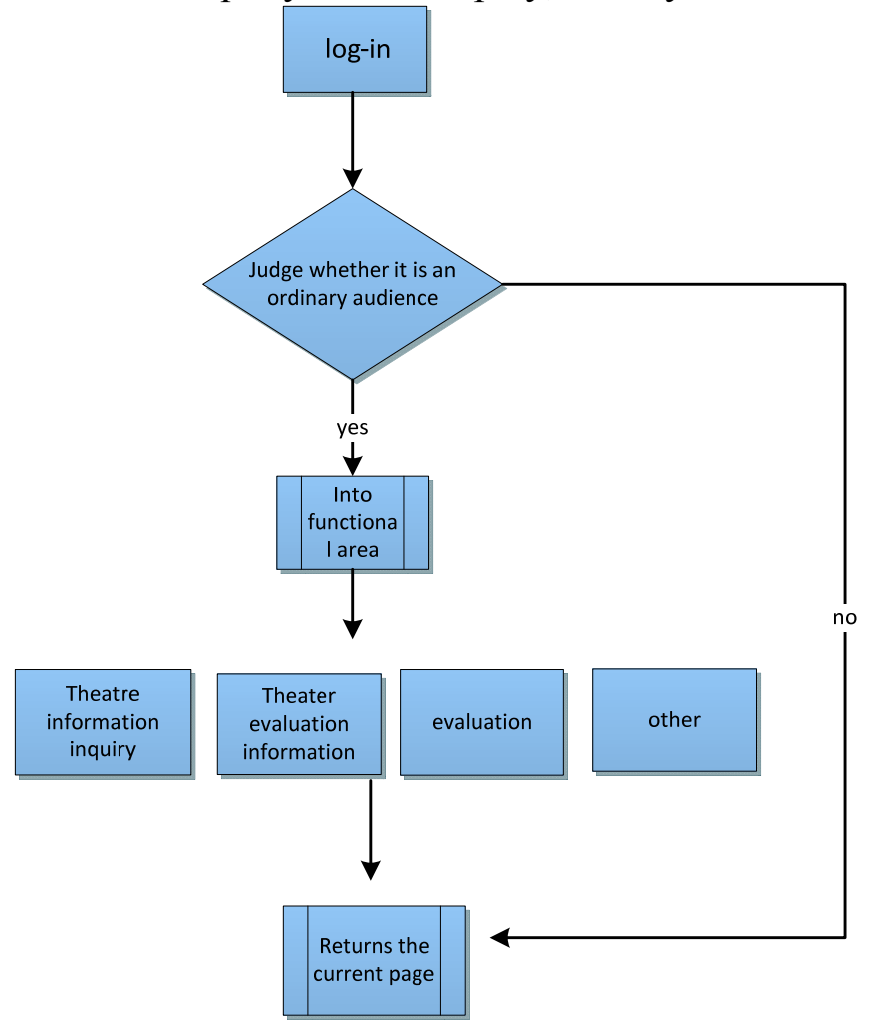

Fig. 1 The process of ordinary audience

\section{B. Expert users}

Expert users includecinema building designers, engineering audit personnel,etc.

Theirlanding process is similar to ordinary users. They have a similar query function with ordinary users, but also with a professional evaluation of the theater function.

The theater is divided into four areas: the acting area, the performance preparation area, the service area and the audience area. In accordance with the evaluation standards, experts rate the elements of the area by one by one. When the expert's ratings are finished by clicking the submit button, the system calculates the score of each area by edited formula, and then we can obtain the total score of the theater. (Figure 2)

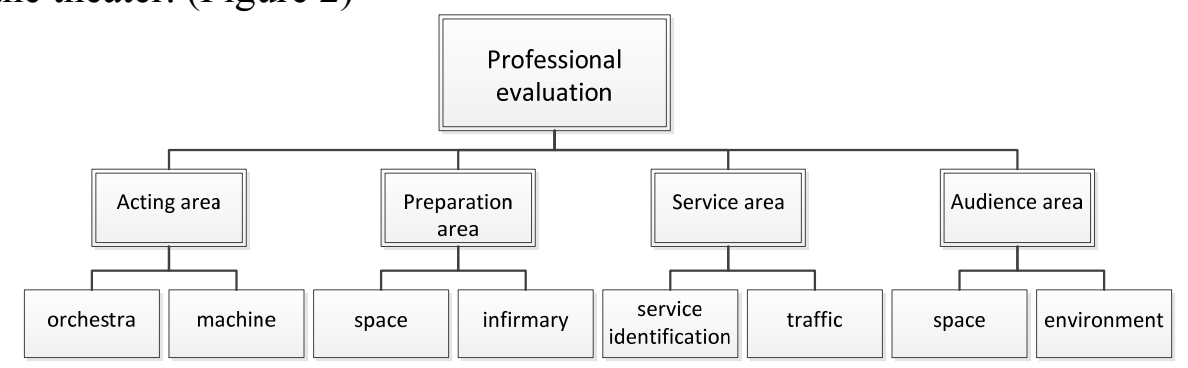

Fig. 2 The professional evaluation 


\section{Administrators}

Administrators manage the whole system, so that they can ensure the normal operation of the system, andensure the safety of the website information.

After entering the system, the administrators can browse information, but the most important is that they have administrative authority. Therefore, they can manage the theater, the users and the information. (Figure 3)Administrators protect the effectiveness and security of the whole system information by adding, modifying and deleting.

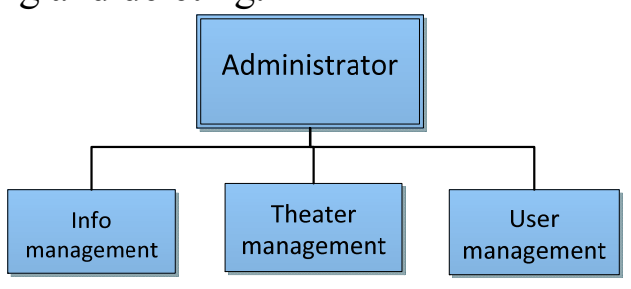

Fig. 3 The function of administrator

\section{System technical proposal}

In order to meet the needs of the rapid development and maintenance of the system, we use the $\mathrm{B} / \mathrm{S}$ frame to build a network platform of theater evaluation.At first, using bootstrap framework under W3C standard to construct the front-end of the website. HTML/CSS/JavaScript are used to be the mainly language to achieve the layout of the page, animation performance and content display. The back-end development uses PHP, the lightweight language, to build a data exchange model. At the same time, MySQL is used as database to store data. The core algorithm uses the method of theater scoring in the model of analytic hierarchy process. Finally, we achieve web browsing through the Apache server and MySQL database to build the server and the database, which are integrated in XAMPP.

A. Front-end development technology

The core step of the development is the application of div +css layout, the bootstrap framework is the classic layout framework ofdiv + css. We define all the elements in the page and topology structure through CSS style sheet. And wewrite inline stylesheet or embed external style sheet in the page, and introduce element attribute in the style sheet in the ways of $<$ div class $=i d=>{ }^{[2]}$

It is required to introduce the CSS file through<link href="css/bootstrap.min.css" rel="stylesheet" media="screen" $>$ in the header file when the system is introduced into the bootstrap framework. Before the introduction of the JavaScript file, the jQuery file needs to be introduced.

(a)BootstrapCarousel

Bootstrap Carousel is a flexible way of responding to the website to add a slider. The content is flexible enough to be an image, an embedded frame, video, or any other type of content. In order to show the image and function of the website, we insert the HD picture of the theatre in the form of carousel, and provide the users with access to the theater entrance connection.

One can add a title to the slide by element carousel-caption inside item in the CSS file, and adjust the position and appearance of the title by $\operatorname{tab}<a>$ and $\operatorname{tab}<h>$.

Loaded pictures can be added to class img-responsive, which could let the picture support the responsive layout. It set up max-width and height and display properties to the picture, in order to make the picture better scaling in the father element. [2]

(b)Functional page

The tag $<$ nav $>$ is introduced into the Sub-navigation list in the functional page, and make cont ent block be adaptive to the middle and justify.

The drop-down box and search buttons use grid system to typeset. The grid system contains a predefined class which is easy to use, and the powerful mixin is used to generate a more semantic la yout. The grid system creates a page layout through acombination of a series of rows and columns.

(c) JavaScript dialog box 
The JavaScript dialog box contains a warning box, a confirmation box, and a prompt box.In the theater evaluation system, we use the warning box to prompt the message. Users need to click the OK button to continue to operate when the warning box appears. [3]

After clicking on the button, you can achieve the operation of the refresh or return by using related instruction of window jump or page jump. Specific code is as follows:

Echo "<script>alert(' User name already exists'); history.go $(-1) ;</$ script $>$ "; $\quad / *$ It warns that the user name already exists. And return to the current page.

Echo "<script>alert (' Verification code is not correct!);window.location. href='register.php'</script $>$ "; /* It warns that the Verification code is not correct. You can use $<$ href $>$ to jump to any new page.

B. Server and database technology

a. MySQL database

MySQL database is a Relational Database Management System (RDBMS) with open source. MySQL database system usesStructured Query Language for database management.Because of its speed, reliability and adaptability, MySQL has received much attention.So, we choose MySQL as the database of this system. [4]

b. Server development

In the W3C system, we demonstrate server-side scripting by using Active Server Page (ASP) and Hypertext Preprocessor (PHP).

PHP is a common open source scripting language. The syntax combines the features of $\mathrm{C}$ language, Java, and Perl. It is conducive to learning, and it is mainly used in the field of Web development. PHP can perform dynamic web pages more quickly than CGI or Perl. Compared with other programming languages, PHP execute program which is embedded into the HTML document, the executive efficiency is much higher than CGI. PHP can also perform compiled code. Compiler can encrypt and optimize code execution, and make code running faster. [5]

Moreover, the biggest feature of PHP is that it is simple and has a natural combination with MySQL.We use function mysql_connect () to complete the connection between the server and the database.

C. The core algorithm of the system

In this system, we use the Analytic Hierarchy Process (AHP) to carry on the professional appraisal to the theater. AHP mainly do the hierarchical analysis of the problem. We obtain the factors affecting the nature of the problem through perceptual knowledge and rational analysis.According to the relationship between the factors and the subordinate relationship, all kinds of factors are combined in different levels. Finally, a multi-level analysis structure model is formed.Then it makes the problem more intuitiveby the quantitative calculation of information.

Analytic Hierarchy Process requires that the hierarchical structure is composed of the following three levels:

Target layer (top layer): Predetermined target of the problem;

Standard layer (middle layer): Guidelines for achieving goals;

Measure layer (lowest layer): Measures to achieve goals.

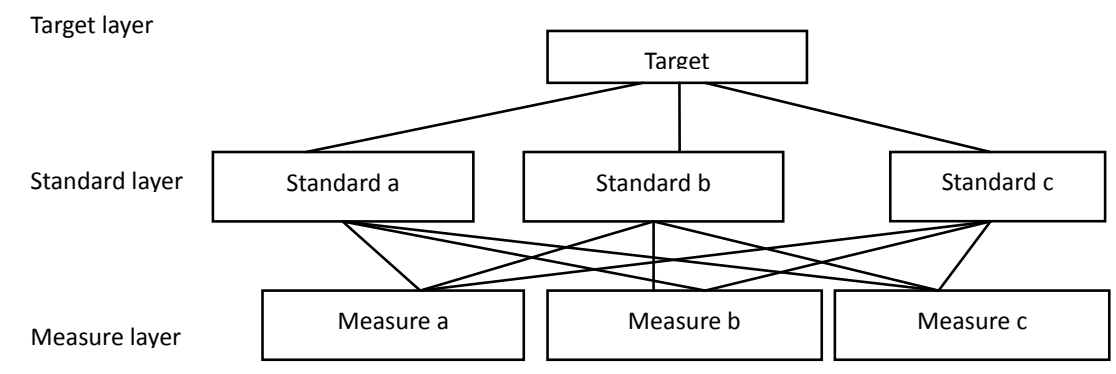

Fig. 4 Hierarchical structure diagram

The target layer of the evaluation system is the whole theater.The next is the standard layer. We divide the theater into four areas: the acting area, the performance preparation area, the service 
area and the audience area, as shown in figure 5. Each area should be divided by the facilities it contains. Then, we should consider the different elements to further divide. Specifically,we take theatre service area as an example. Andwe can get the scoring system of the service area according to the analytic hierarchy process. (Table 2 )

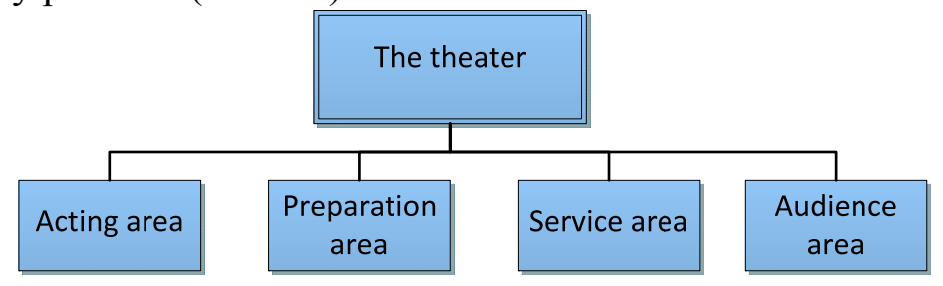

Fig.5Theatre Division

Tab. 2the scoring system of the service area

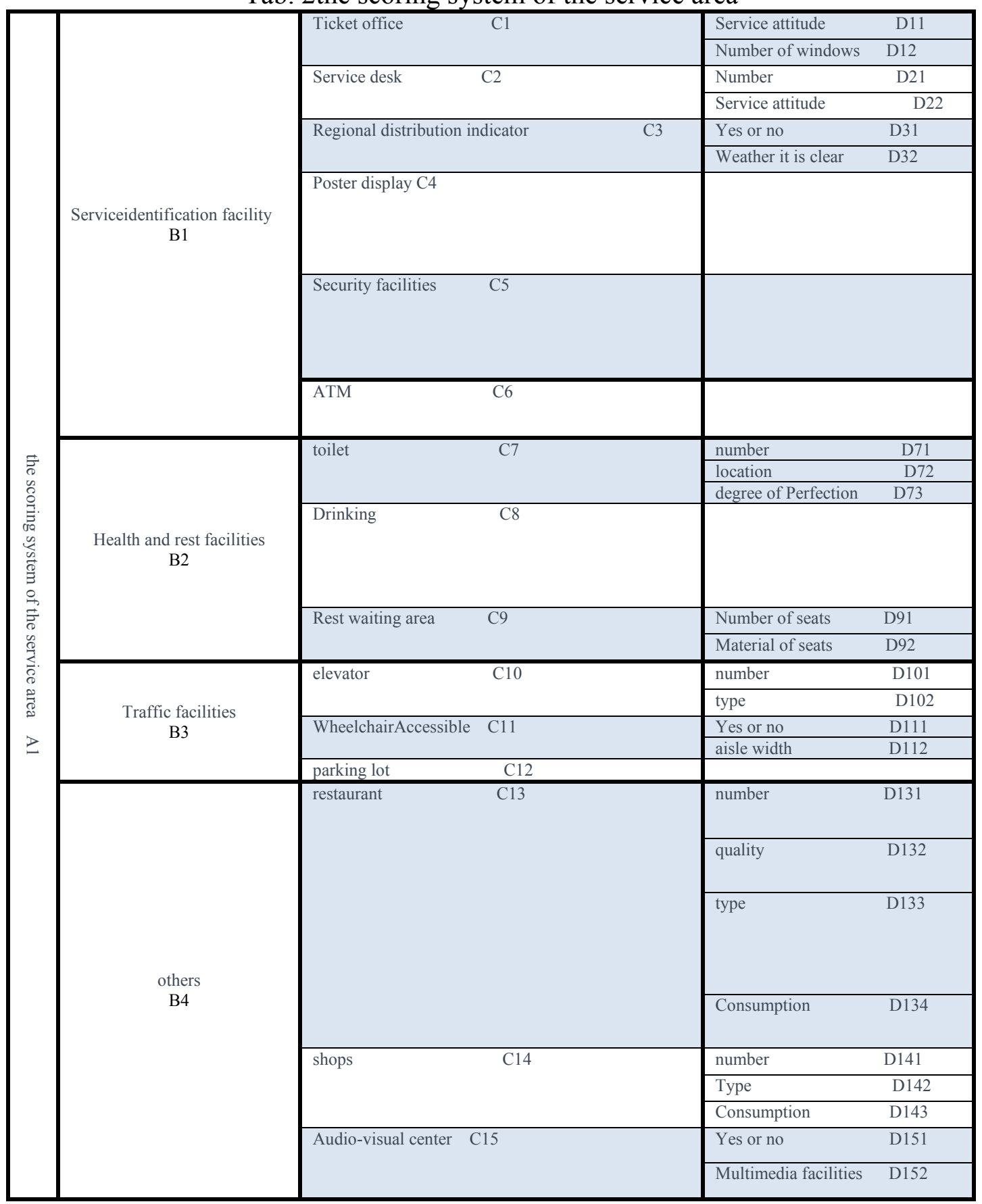

After establishing the scoring system, we analyze the different factors of each area to get the weight of each factor. And according to the weight, we can use PHP and database technology to 
calculate the corresponding fraction and proportion.

Concretely, according to the AHP, an expert scores each factor of three theaters. And after the calculation of the system, we can get the total score of the theater and the score of each area. For example, an expert named zhuanjial scored Guangzhou Theater, Chongqing Theater and Shanghai Theater. As is shown in figure 5 and figure 6, we can lay the conclusion that Shanghai Theater is the best theater of the three theaters.

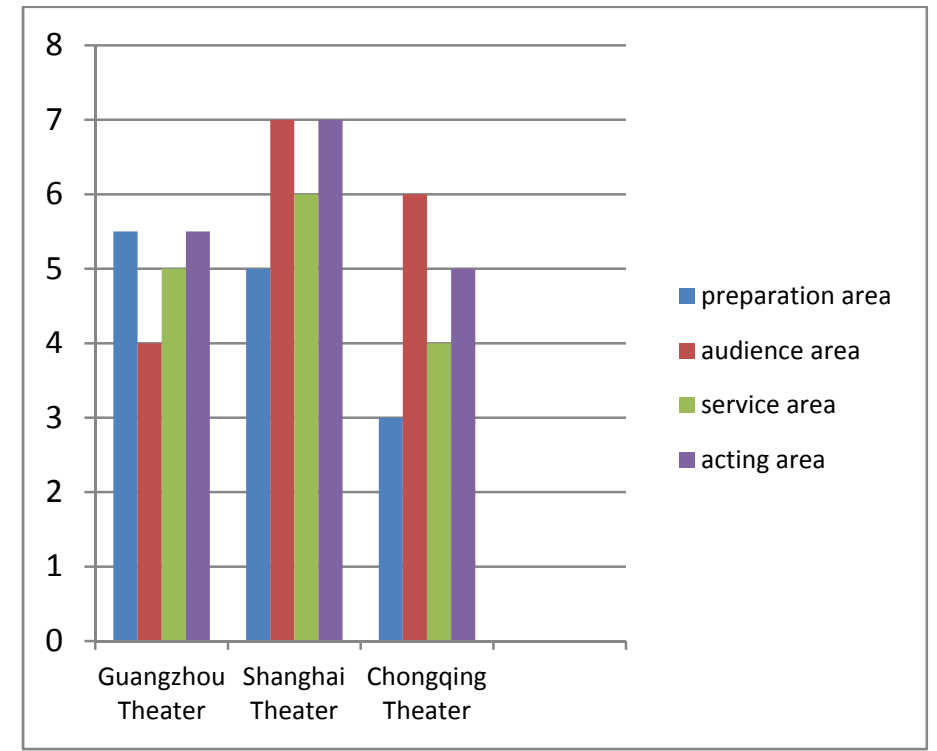

Fig. 5 the score of each area of the theater

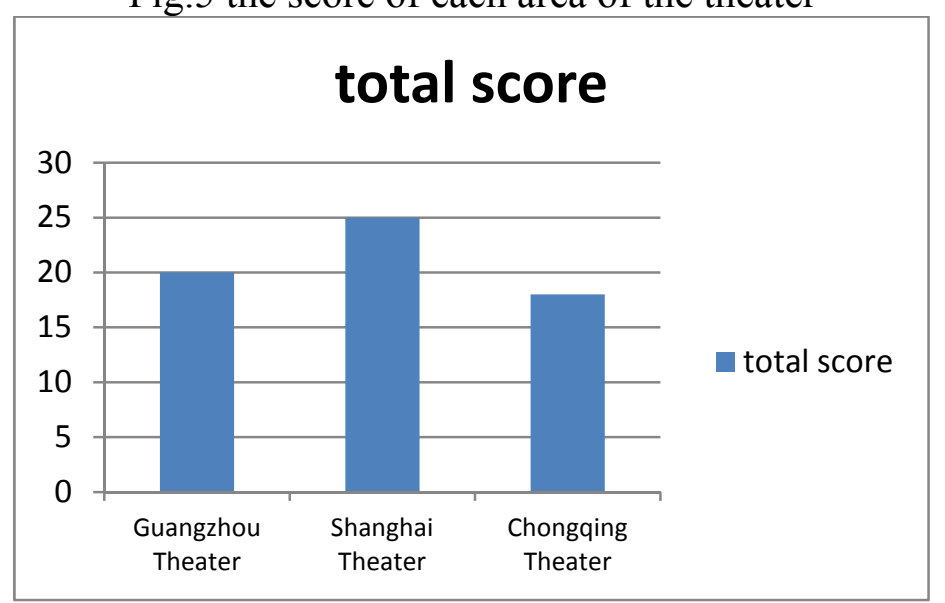

Fig. 6 the total score of the theater

\section{Summary and Outlook}

The evaluation system of the theater is built to collect information of the theater, at the same time, it shows theater evaluation information for more audiences, performers and builders through the users' experience and the expert's professional evaluation. The personal experience of the audience can provide consumption reference for the consumers.Experts' advices and opinions, to a certain extent, could improve the construction 1 and $t$ operationlevelof the theater. So the perfect effect of the performance can be achieved through the coordination of facilities and manpower. Andit can provide the audience with high quality and attentive service. On the other hand, the integration of information can realize the dispatcher and management of cultural performances resources, and provide technical support for the collaborative arrangements of cultural performance schedule and the cooperative scheduling of performance resource.

This system integrates the system space with the audience, the experts in the field and the administrator.In the design, we use the bootstrap framework for development, and use HTML, CSS and JavaScript for layout beautification. Meanwhile, PHP language algorithm is used to achieve data exchange with the MySQL database. Finally, webuild an intuitive and convenient interactive 
website for the users. Although, from the perspective of website construction, it is not perfect, the construction of this system is of great value and prospect from the perspective of the theatre construction and market demand.With the rapid development of the contemporary cultural industry,this kind of information system is essential for the development of the market which integrated information browse, consumption reference and professional evaluation. Moreover, on the basis of meeting the needs of the audience and providing the standard of theatre construction, the system can promote the development of the theatre, improve the service level and strengthen the cultural construction of our country.

\section{Acknowledgement}

We would like to thank the National Science and Technology Support Program (Foundation project: Research on interface protocol of Stage manage, Project number: 2012BAH38F01-04)and Engineering Project of CUC (Foundation item: Research on data interaction Vision-Audition stage management system, Project number: 3132015XNG1530) for their help and support.

\section{References}

[1]Weiping Liu," My view on the construction of Chinese Theatre” J. Nanjing daily.Aug 2013

[2]Runood.com. "Bootstrap carousel tutorial”.[EB/OL]

[3]RonglingGao," The advantages of Bootstrap in the development of the front-end" J. Computer CD software and Application. Jan 2015

[4]Yi Lv," JavaScript framework to solve the problem of browser compatibility in the development of WEB program" J. Technology rich guide. Dec 2010

[5]Yapeng Li, "Research of data management based on MySQL database" J. Times of Science. 2013.

[6]EN science and technology," The difference between ASP and PHP in the website construction".[EB/OL] 